\title{
MIGRACIÓN, DIVERSIDAD Y EL AULA ESCOLAR
}

\author{
Éricka Vásquez Salazar' \\ Somos seres históricos. Esta afirmación ha sido \\ hecha y no representa ninguna novedad. Pero vale \\ la pena insistir en su sentido: somos producto de \\ experiencias anteriores, de conocimientos, vivencias, \\ tecnologias, cultura, aciertos, errores, violencia, \\ encuentros y desencuentros atesorados a lo largo \\ de generaciones. Somos producto de esa historia \\ general y de nuestra biografia, de la manera en que \\ lo general es vivido en nuestra vida cotidiana.
}

Gutiérrez y Prieto (1991, p. 34)

\section{Resumen}

Las migraciones se presentan como una constante histórica, que han permeado en gran medida lo que somos hoy. En Costa Rica, se reciben miles de migrantes al año, especialmente del país vecino Nicaragua. Su presencia ha logrado transformarnos social y económicamente, pero sobre todo ha tenido una gran influencia en el ámbito escolar. Todo docente debe, por lo tanto, estar preparado para atender a una población diversa, esforzarse por brindarle una educación de calidad que tome en consi- deración las características de todos los actores involucrados en el proceso. Para lograr lo anterior, deberá considerar aspectos sociales, económicos y emocionales, entre otros, y buscar incluir, constantemente, en el currículo elementos que ayuden a insertar a los estudiantes migrantes, para darle significatividad al proceso de enseñanza y aprendizaje. El respeto por lo diverso debe rescatarse y hay que hacer un esfuerzo por lograr un acercamiento más profundo al otro y a sus diferencias, y transformar el aula en un espacio donde haya lugar para cada estudiante.

1 Académica de la Universidad Nacional. Máster en Pedagogia con Énfasis en Diversidad en los Procesos Educativos, de la Universidad Nacional. Licenciada en Docencia, de la Universidad Estatal a Distancia. Actualmente es Subdirectora de la División de Educación Básica del Centro de Investigación y Docencia en Educación. Recibido: 2 de noviembre-2006 - Aprobado: 30 de noviembre-2006 
Palabras claves: migraciones, diversidad, aula escolar, currículo, escuela, docentes.

\section{Abstract}

Migrations seems to be an historical constant that have influenced what we are today. Costa Rica receives thousands of immigrants every year, especially from the neighboring country, Nicaragua. Their presence has transformed our economic and social reality, but mainly it has had a great influence in the dynamic of educational institutions. Therefore, school teachers must be prepared to attend a diverse population, making an effort to offer an education of quality that takes into consideration characteristics of all the actors involved in the process. In order to achieve this, it will be necessary to consider social, economic and emotional aspects, as well as to work for the addition of different elements in the curriculum that allow migrant students to be inserted by providing them a meaningful learning process. Respect for diversity must be achieved by means of an effort to supply a deeper view of the other and its differences, transforming the classroom in an inclusive space for all students.

Keywords: migrations, diversity, classroom, curriculum, elementary school, teacher.

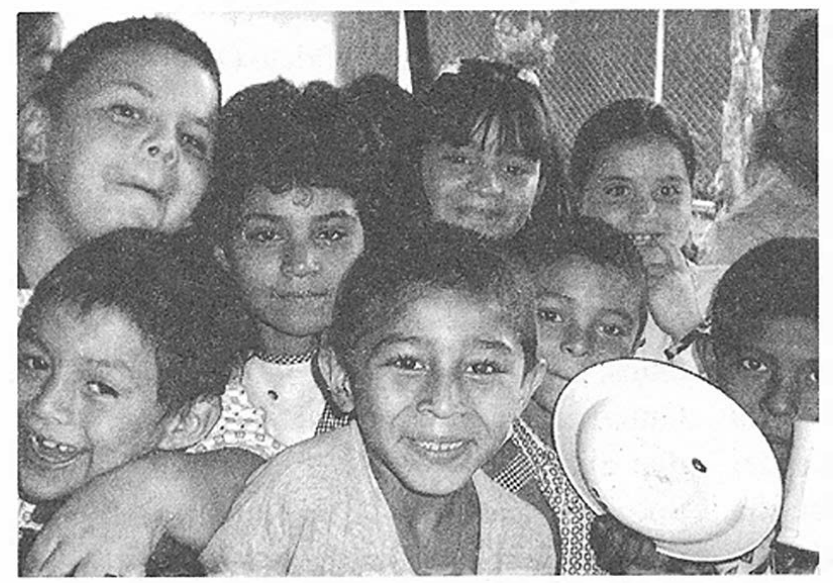

Zl hombre y la mujer, como Useres históricos, están conformados por múltiples componentes, la migración es uno de ellos. El fenómeno migratorio es inherente al ser humano, ha sido parte de los procesos de conformación social a través de los siglos, y es parte de la globalización que se vive en la actualidad. Así lo mencionan Barth y Díaz (2000) al decir que los movimientos migratorios constituyen uno de los hechos humanos de todos los tiempos, y son los que han conformado la humanidad tal y como es hoy, y le han dado la gran diversidad cultural que posee el ser humano en todos los rincones del planeta.

Las causas que provocan dicha tendencia migratoria en el ser humano son múltiples y han ido variando con el paso del tiempo. Antiguamente, las exploraciones e incursiones en territorios nuevos, tal y como sucedió en América, 
provocaban la colonización y conquista de las nuevas tierras, trayendo consigo oleadas de migrantes que buscaban riquezas y nuevas oportunidades de vida. En el siglo pasado y en el presente, la migración ha sido consecuencia de guerras, crisis políticas en diferentes paises, del desencadenamiento de la problemática económica mundial y de la globalización.

Barth y Díaz (2000) comentan que dentro de los grupos de migrantes podemos encontrar a aquellas personas que se ven obligadas a vivir fuera de sus paises por diversos motivos, tales como los políticos, ideológicos, religiosos, y otros; todas estas personas esperan la oportunidad de volver a su patria cuando la situación que provocó su salida se haya modificado; también, las razones económicas son de bastante peso para que las personas decidan salir a trabajar temporalmente en otro país, con la idea de volver en un plazo determinado.

Como ejemplo de lo anterior, se puede mencionar la gran migración de suramericanos y suramericanas que a causa de las diferentes crisis políticas sufridas en países como Argentina y Chile, durante la segunda mitad del siglo pasado, debieron exiliarse como forma de supervivencia ante lo acontecido y convertirse en migrantes que, muchas veces, añoraban con volver a sus países de origen. "Brasil con 1.5 millones y Colombia con 1.9 millones de personas, son los países. de la región con mayor número de emigrantes al año" (Ażofeifa, Monge, Vidal y Céspedes, 2001, p. 36).

También se da la migración permanente de grupos de personas provenientes de todo el mundo hacia los Estados Unidos en búsqueda de mejores oportunidades de vida. Según Porter y Romo (1999, p. 37), "el año pasado 8.000 agentes de la patrulla fronteriza de los Estados Unidos detuvieron a 1,4 millones de inmigrantes indocumentados, la mayor parte de ellos mexicanos... lo cual no evitó la entrada de 275.000 nuevos indocumentados que se sumaron a los millones ilegales que viven en el país".

En Europa, a causa de cambios políticos acontecidos en años pasados, se desataron luchas internas que obligaron a las personas a migrar desde sus lugares de origen, en calidad de refugiados y refugiadas hacia diferentes países aledaños, entre estos citamos a los ciudadanos de Kosovo, Serbia, Croacia y Albania.

En el Medio Oriente, las migraciones se han intensificado paralelamente a los conflictos bélicos de la región; Afganistán e Iraq son ejemplos claros de cómo en determinados momentos históricos, grupos numerosos de personas, en algunos casos villas enteras, deben emigrar como respuesta a una amenaza a su vida. 
Todas estas migraciones, que han sido y serán una constante histórica en el desenvolvimiento del ser humano, no deben ser ajenas. El mundo se ha transformado en un lugar donde las fronteras y los límites han dejado de ser tan importantes, por lo cual la historia por sí misma exige que se mire lo que acontece alrededor. En palabras de Savater (2001), se debe llegar a tomar conciencia de que pertenecemos a una misma especie, la humanidad y que, juntos en una visión mucho más allá de lo biológico, debemos apuntar a un proyecto común, a una forma de comprender lo humano desde una fraternidad básica. Este autor señala que la indiferencia no cabe dentro de nuestras apreciaciones, ya que la humanidad del otro siempre compromete la de los demás.

Tomando en cuenta la realidad del fenómeno migratorio, es momento de darse cuenta cuán comprometido se está. Según estudios realizados por la Organización de las Naciones Unidas a inicios de la década de los noventa, más de 100 millones de personas vivían fuera de su país de origen; según datos de Barth y Díaz (2000), de estos 30 millones eran trabajadores extranjeros y trabajadoras extranjeras y 23 millones personas refugiadas.

Costa Rica, específicamente, es un país que dentro de sus procesos históricos, tanto externos como internos, ha contado con movimientos migratorios importantes que han ayudado a construir su sociedad, y al enriquecimiento étnico-demográfico que posee en todas sus áreas. Debido a su posición estratégica, ha sido puente para muchos migrantes hacia otros países. Así como también, por sus características políticas y sociales, se ha convertido en la tierra prometida para muchos otros centroamericanos que buscan un lugar mejor donde vivir.

Con referencia a lo anterior, Costa Rica, según Acuña y Olivares (1999), se ha caracterizado históricamente por ser un país receptor de población migrante. Es así como, el abordar las temáticas de diversidad y migración se presenta como un reto y una responsabilidad para todo educador, ya que entender esta diversidad, que actualmente se presenta en las aulas, es importante para desarrollar una labor docente de calidad; para ello, es necesario

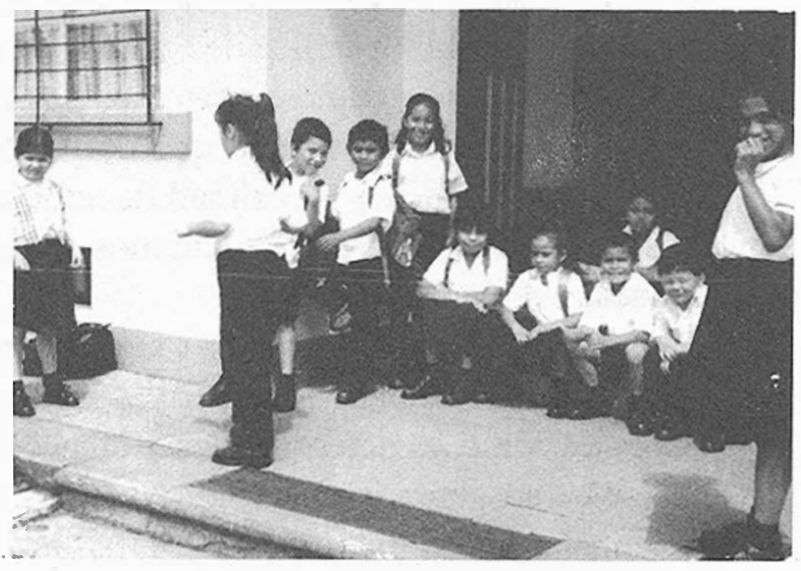


comprender que actualmente el sistema educativo costarricense debe proveer educación a miles de niños migrantes que ocupan las aulas. Este reto se ha ido intensificando con el tiempo y continuará haciéndolo, tal y como lo mencionan Contreras y Robert (2000), en el sentido de que los y las estudiantes de origen nicaragüense aumentan un $25 \%$ como promedio cada año desde 1990.

Esta realidad puede ser abordada desde múltiples perspectivas, pues se tienen a disposición muchos estudios que muestran estadísticas que tratan de describir numéricamente la realidad que se vive. También los medios aportan una cuota de notas en las cuales se habla del migrante en nuestro país y las consecuencias que esto tiene; pese a lo anterior y aunque las cifras sean alarmantes por sí solas, tal hecho no es suficiente para lograr comprender la situación de los migrantes que viven en el país, ya que esta es una realidad que requiere ser vista con una mirada cercana y humana.

Lo primero es aceptar la relevancia de la migración nicaragüense hacia Costa Rica, y su importancia en la conformación histórica de nuestra sociedad, lo cual lo convierte en un fenómeno de años de existencia, inherente a nuestro vivir cotidiano. Una relación constante marcada por factores políticos y fenómenos naturales es lo que ha impulsado la migración de nicaragüenses a suelo costarricense, en busca de una mejor calidad de vida para ellos y sus hijos.

A pesar de que han habido múltiples esfuerzos para que Nicaragua reactive su economía, esto no se ha logrado; aún menos ha logrado una reactivación social. Por lo contrario, ha provocado que haya empleo, pero con sueldos muy bajos, que las condiciones de vida no mejoren, que continúe habiendo carencias en salud y educación, sobre todo en los sectores de clase baja. Los y las migrantes no han sentido una mejoría en su situación, sino más bien esta ha tendido a empeorar.

Lo anterior ha impactado, de forma considerable, la educación en el país, pues la presencia de esta población ha cambiado el panorama en muchas de las instituciones educativas. Mata, Monge, Pereira, Alvarado, Gómez, Cubero y Frías (2000a, p. 28) han estimado que:

"El esfuerzo educativo que se haga, a la par de otras acciones de carácter económico y social en general, permitirá que se alcance, una vez más, la utopía de un desarrollo social armonioso. Para ello, se debe buscar la atención de las necesidades con que se integran las comunidades nacionales los inmigrantes centroamericanos que dejaron sus tierras primero 
amenazados por guerras internas, después por los acontecimientos naturales que impactaron sus países y que destruyeron temporalmente sus sueños. Las escuelas costarricenses han abierto sus puertas a todos aquellos niños y niñas que habitan en el país sin importar sus lugares de nacimiento".

Tal y como lo citan los autores y las autoras, las puertas se han abierto, pero es mucho más real afiontar el fenómeno desde todas sus perspectivas, como se ha mencionado; donde la base educativa en valores es un eje primordial sobre el cual apoyarse, y como premisa se debe colocar a la vista la problemática que sufre el estudiante migrante, luego de que cruza la puerta que se le ofrece.

Según un estudio realizado por Vásquez (2004), estos niños viven en un contexto con características sociofamiliares muy complejas y peculiares, que se caracterizan, entre otras, por vivir el ingreso a Costa Rica como una experiencia de temor, hambre y malos recuerdos, tener padres y familiares que se emplean en labores agrícolas, servicios domésticos, seguridad, construcción, empacadoras, con jomadas extensas de trabajo, habitan en casas pequeñas que no cuentan con los servicios básicos.

También, se logró ver que su desempeño en el ámbito escolar se ve afectado por factores como analfabetismo de padres, falta de oportunidades para desarrollarse plenamente en el aula, rechazo y sentimientos de inferioridad y no pertenencia, sentimientos negativos hacia el estudio y la escuela en general, ausentismo, baja autoestima, extraedad, rezago y desnivel y poco tiempo para estudiar en el hogar. Lo anterior es parte de la realidad del niño migrante y, por lo tanto, le afectará de forma negativa en su proceso de enseñanza y aprendizaje.

La situación particular del niño migrante provoca que este se encuentre en una situación de desventaja en comparación con el y la estudiante costarricenses, que además de no vivir la problemática que acarrea el traslado de un país a otro, también ha sido educado en el sistema educativo costarricense desde sus primeros años escolares; cada nivel cursado ha estado caracterizado por los estándares del currículo del Ministerio de Educación Pública y, en la mayoría de los casos, la edad no corresponde a la que deberían tener según el nivel que cursa.

Esta situación es de desventaja y provoca que estos niños y estas niñas en la escuela presenten un bajo rendimiento escolar; según Mata et al. (2000a, p. 30), los principales factores que afectan el rendimiento del niño migrante en relación con lo anterior son los siguientes: 
"El rezago escolar, producto de una formación insuficiente o de problemas de aprendizaje que impiden al educando cumplir con los estándares establecidos para los niños y niñas de edades similares que cursan un determinado nivel educativo. La extraedad, manifiesta no en términos cuantitativos sino entendida cualitativamente como las dificultades psicopedagógicas que sufre la población estudiantil de mayor edad que la establecida por el sistema escolar, y que por lo tanto puede generar problemas de rezago escolar. La desnivelación, que se refiere a los casos en que los alumnos y alumnas migrantes ingresan a un nivel específico del sistema educativo, inclusive con la documentación que los acredita, pero con un nivel académico inferior al nacional".

Como se comentó anteriormente, no solamente lo académico repercute en el rendimiento. Según James (1997a), los niños migrantes de hogares pobres tienen mayor propensión a tener problemas de salud, depresión, baja autoestima, agresión y problemas académicos.

En esta línea, se pueden agregar algunas características psicosociales encontradas en estudiantes migrantes (Vásquez, 2004), como son problemas al interactuar con sus compañeros, sufrir constantes burlas y sobrenombres, ser rechazados por el grupo y hasta por los docentes, sentirse aislados en el aula y esconder tanto su nacionalidad como su forma de hablar.

Según James (1997b), el estudiante sufre de falta de aceptación por parte de sus compañeros y muchas veces por parte de sus maestros, lo cual lo lleva a sentirse diferente y con un sentido de ausencia de pertenencia a la realidad actual. También, le afecta en demasía la visión de la sociedad del grupo étnico al que pertenece, lo cual al ser negativa llevará a la discriminación y rechazo, y hará que se sienta deprimido, confuso, desesperanzado y fuera de lugar.

Muchos estudiantes migrantes tienen problemas relacionados con la identidad y con el hecho de lograr asimilar la vida escolar y la vida en la casa, las cuales varían enormemente, según lo comenta Perkins (2000). Respecto a esto, James (1997b) menciona el hecho de que la diferencia entre los valores del país de origen y familiares con los del país al cual inmigran representan también un obstáculo para el aprendizaje y un factor de riesgo. Asimismo, muchos de estos niños sienten el temor de ser ridiculizados o discriminados al revelar su lugar de procedencia y sus costumbres.

Estos hechos pueden ser descritos como un choque cultural que sufre el estudiante migrante y le afecta en todos los ámbitos de su vida, pero mucho 
más en el educativo, ya que será el lugar donde establecerán el contacto con la realidad de la nueva cultura y se percatarán del cambio en su entorno. En la opinión de James (1997a, p. 9):

"El choque cultural es precipitado por la ansiedad del resultado de perder todo signo familiar y símbolo de interacción social. Dichas pérdidas producen tensión, intranquilidad y hasta desajuste emocional si la persona es recibida negativamente. Algunos niños enfrentan esto sin la presencia reconfortante de sus padres o hermanos porque algunos niños son enviados a vivir lejos con parientes o uno de los padres se queda atrás. En las familias de migrantes de bajos ingresos puede ser difícil para los padres nutrir a sus niños totalmente, ya que la experiencia tratando de encontrar trabajo o trabajar por largas horas también desgasta la energía de los padres".

Por otra parte, el clima de hogar es sumamente importante a la hora de determinar el desempeño del estudiante en el proceso de enseñanza y aprendizaje. Respecto a esto, Mata et al. (2000b) mencionan el clima hogareño entre las características tales como oportunidades de juego, calidad de la vida familiar en cuanto a relaciones padre y madre, su influencia sobre la seguridad del niño y el desarrollo de su personalidad en general y la condición económica de los padres.

Según Mata et al. (2000c), un elemento que afecta negativamente el éxito escolar del niño inmigrante es la falta de respeto por la diversidad en el aula. Estos autores comentan que es de suma importancia tener en cuenta las diversas características culturales de los grupos presentes en el momento de llevar a cabo las actividades curriculares. Mencionan tres factores que evidencian las diferencias entre culturas distintas: el tipo de organización que tienden a construir las personas de diferentes grupos culturales, sus estilos de aprendizaje y sus estilos convencionales de comunicarse, la sociolingüística.

El primer factor está relacionado con la forma de trabajo, ya sea individual o en grupo; el segundo, con el entorno del niño y las características culturales y socioambientales en las cuales se desenvuelve $\mathrm{y}$, por último, el tercer factor, con las normas de urbanidad y los convencionalismos culturales que se demuestran mediante el lenguaje. Mata et al. (2000c, p. 8), en la misma línea, agregan que: 
"El éxito escolar y la integración social se alcanzan en gran medida, cuando los y las estudiantes comprenden cuándo, dónde y cómo comunicarse. No puede esperarse que todo el grupo conozca de antemano qué tienen que hacer, cómo deben comunicarse entre sí y con los docentes o las docentes y que sepan resolver acertadamente las situaciones conflictivas que requieren de la comunicación de sentimientos".

Esto muestra lo compleja pero enriquecedora a la vez que es la naturaleza misma del migrante, y que los criterios superficiales no darán una imagen real y fidedigna de lo que en realidad hay en lo más profundo de la población migrante, tanto en lo colectivo como en la individualidad.

Por esto, y más allá de una estadística, debemos entender que los niños migrantes, así como sus familias, deben afrontar no sólo las necesidades económicas, sino también la pérdida de una vida como la conocían hasta ahora, el dejar atrás a familiares queridos, el tener que separar la familia que antes estaba unida, el abandonar el rancho con todo lo que tenían, el olvidarse del paisaje que les resultaba familiar, de no transitar más por los mismos caminos

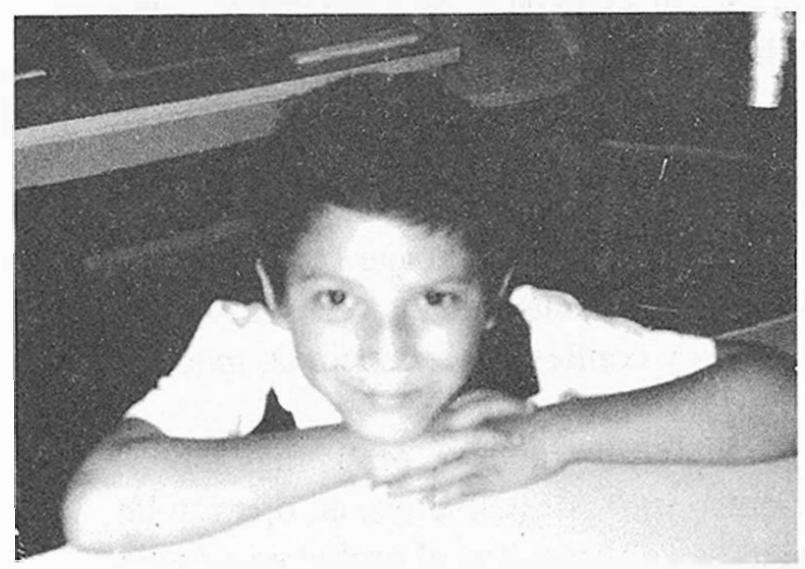
ni volver a saludar a los vecinos y a las vecinas; todo esto aunado al hecho de que deben insertarse en una sociedad que no los comprende, ni los quiere, sociedad llena de terribles estereotipos y prejuicios.

Lo anterior debe ser vivido sin poder tomarse el tiempo para entender qué es lo que está sucediendo; los mayores porque deben trabajar y conseguir un lugar donde vivir, los más pequeños porque deben ayudar con la nueva vida que se está forjando y saben que no hay tiempo para lamentarse, simplemente la orden es sobrevivir. Sobrevivir, en la mayoría de los casos, de forma precaria, donde muchos viven en condiciones de pobreza extrema, en hacinamiento, con escasez de comida y, muchas veces, sin los servicios básicos necesarios para llevar una vida digna.

En este panorama, es de suma importancia conocer la realidad de quienes ocupan un espacio en el aula; además, se debe ir más allá y tratar de brindar más 
que conocimientos, que en muchas ocasiones carecen de significado para el estudiante, así como ofrecer un lugar donde este pueda desarrollar su potencial y sienta que está protegido y aceptado.

Perkins (2000) menciona que los rápidos cambios en la población estudiantil plantean numerosas cuestiones a los y las docentes; aquí es donde lo esencial es comprender la cultura y experiencias de estos niños, para lograr una experiencia exitosa.

Es indispensable recordar que los niños tienen obligaciones que los protegen, y que de esta manera el educador está en la obligación de brindarles la atención que los y las estudiantes inmigrantes requieran; Mata et al. (2000a, p. 47) mencionan que:

"El maestro y maestra debe recordar que todos los niños y las niñas sin distinción alguna (por motivos de raza, color, sexo, idioma, religión, opinión política, origen nacional o social, posición económica, nacimiento, impedimento físico, realidad o necesidad particular o cualquier otra condición), tienen los mismos derechos y libertades, los cuales son inviolables, ningún niño o niña será privado de ellos, sean costarricenses o extranjeros, estén en condición legal o ilegal, sus derechos deben cumplirse".

Perkins (2000) comenta el hecho de que muchos educadores y muchas educadoras no tienen la capacitación para enfrentar el trabajar con otras culturas, lo cual constituye todo un reto. Este obstáculo algunas veces impide la adecuada atención de los niños migrantes y conlleva al irrespeto de muchos de sus derechos.

Habiendo citado el rezago y la extraedad como los problemas fundamentales, un problema más que enfrentan dichos estudiantes, en opinión de Mata et al. (2000a), es que el o la docente debe resolver el problema de aula que genera el hecho de que los niños han pasado largos períodos sin instrucción escolar o han sido preparados bajo diferentes currículos que exigían menos que el costarricense. Esto trae consigo la necesidad de atención especial para el niño o la niña, quien necesitará que se le cubran áreas como las sociales y las afectivas, entre otras.

En este mismo sentido, James (1997b) plantea que el personal de la escuela deberá entender los factores y circunstancias que afectan el mundo interno del niño migrante, y cuando lo hace, tiene que decidir si diferentes 
metodologias o propuestas son necesarias, o si usa las herramientas existentes con diferentes metas. Los programas deberán contener temas como el estrés de perder su cultura y ganar otra, la falta de habilidades sociales efectivas y comportamientos en la escuela que pueden ser incongruentes con las expectativas del estudiante migrante.

Asimismo, el docente debe considerar las problemáticas tanto psicosocial como psicoafectiva que afectan al niño migrante y que claramente tendrán estrecha relación con su rendimiento escolar; al respecto, Mata et al. (2000a, p. 37) opinan que el maestro deberá,

"Tener conciencia acerca de los problemas relacionados con el hambre, la desnutrición y la enfermedad; crear espacios en donde la violencia no exista y predetermine el éxito de los y las escolares; considerar aquellos problemas familiares, ambientales y de recursos culturales a la hora de asignar una tarea, evitar que los prejuicios y los estereotipos medien en la calidad de las relaciones entre compañeros y compañeras de la escuela, entre maestros, maestras y padres de familia".

Es indispensable que el o la docente provea un ambiente en el que sus estudiantes puedan ser constructores de su propio conocimiento y, de esta manera, enriquezcan al grupo con sus experiencias previas. En cuyo caso, el migrante tendrá un espacio para compartir con los otros la perspectiva del mundo desde sus vivencias, así como también le brindará al grupo la oportunidad de comprender un poco más al niño migrante y de compenetrarse con su realidad.

En relación con este tema, Mata et al. (2000c, p. 8) citan que "cl conocimiento por parte de los y las docentes del significado de las diferentes expresiones verbales de sus alumnas y alumnos, favorece la creación de mecanismos de comunicación más efectivos en clase". Asimismo, comentan que es indispensable la sensibilidad y la capacidad que tenga el y la docente para analizar las diferencias culturales y utilizar dichas diferencias para el enriquecimiento del grupo usando una perspectiva de educación multicultural.

No omito mencionar que, bajo cualquier premisa, la diversidad es muy dificil de asimilar en su totalidad y mucho más cuando están en juego intereses personales y el bienestar social. Para poder aceptar de una forma completa a los y las migrantes, entender sus problemas y poder brindarles una educación de calidad, es indispensable un cambio profundo y vinculado con 
los sentimientos propios hacia los otros y las otras, es necesario que sea un proceso vivencial.

Desde esta perspectiva, se deben implementar propuestas que brinden a los y las docentes una opción real de transformación de su visión, a partir de una reconstrucción muy íntima de lo que sienten, creen, piensan y expresan de los y las migrantes. Dicha transformación ha de llevarse a cabo primero, en el docente y, luego este será el encargado de mediar en sus estudiantes y poner en práctica situaciones dentro del aula que lleven a una mejor convivencia y que mejoren la calidad de vida tanto de los migrantes como de los otros niños y de las otras niñas.

De acuerdo con lo anterior, se puede resaltar la importancia de la justicia en la educación, entendiendo justicia no como la igualdad de oportunidades para cada uno sin distinción, sino como el brindar las oportunidades a cada uno en la medida que su cultura lo necesite, considerando las diferencias y rescatándolas en beneficio del bien común.

Es pertinente rescatar que se debe abogar por un modelo de currículo flexible y cambiante, tan cambiante como la cultura misma, que no se queda estático en ninguna de sus manifestaciones. Un modelo que contemple las necesidades de cada persona y le ofrezca al individuo una oferta pedagógica que englobe la identidad como algo que se construye conforme pasa el tiempo, individual y grupalmente, con mezclas y combinaciones que sólo pueden producirse en la cotidianeidad y de la cual la escuela forma parte de ella.

El estudiante migrante está presente en las aulas. En la actualidad, esta es una realidad que no se puede olvidar ni negar. Por el contrario, requiere de toda la atención que sea necesaria para darle la oportunidad de insertarse en la sociedad con la cual hoy convive y, sobre todo, construir las bases para un futuro mejor que el que hoy se le presenta. Desde esta perspectiva, el aula escolar, junto con el estudiante migrante, no deben ser vistos como un problema, sino como un reto para el y la docente que, por la naturaleza de su profesión, deben velar porque sus estudiantes, no importa del lugar donde procedan, tengan la oportunidad de un proceso de enseñanza y aprendizaje acorde con sus necesidades y con el entomo al cual pertenecen.

Para estos niños y estas niñas, que forman parte de la amplia gama de la diversidad que se tiene en las aulas, no es suficiente con ofrecerles una silla y una mesa, en el mejor de los casos, debe brindárseles ayuda integral que englobe lo académico, lo social y lo afectivo para que realmente pueda decirse que están teniendo acceso a una educación digna y eficaz. En cuanto a esto, López (s.f., p. 21) agrega: 
"El discurso de la escuela pública y de la diversidad, que es el discurso de la Calidad de Vida, es un discurso preferentemente ético. Es un discurso que penetra en lo más profundo del ser humano (en la moral), es otra manera de entender al ser humano, y por tanto, a la sociedad. La cultura de la diversidad y la Calidad de Vida es un discurso que exige a la escuela pública que introduzca una nueva axiología y un nuevo mundo de valores, donde las personas han de ser respetadas por eso mismo, por ser personas, y no por el lugar que ocupen en la sociedad ni por el nivel de producción. Sólo así se entenderá cuando afirmo que ser diferente es un valor y no un defecto".

Es necesario, por lo tanto, que el educador asuma una postura de lucha contra la injusticia y la falta de atención que están sufriendo en este momento miles de niños en el aula y, sobre todo, de lucha contra la ignorancia que provoca heridas irreparables con el tiempo; una lucha por el respeto a una calidad de vida que resalta las diferencias.

Por lo anterior, la visión de la diversidad debe pasar de ser un actor pasivo en los salones de clases y llegar a ser contemplada como una variable en las actividades de cada día, dentro de un marco de la comprensión, la tolerancia y la empatía para aquellas personas que hoy comparten con nosotros este país. Como se cita en Alvarenga (1997, p. 53), "uno como ser humano tiene derecho a un pedacito en cualquier rincón de la tierra". Con más razón, pue-

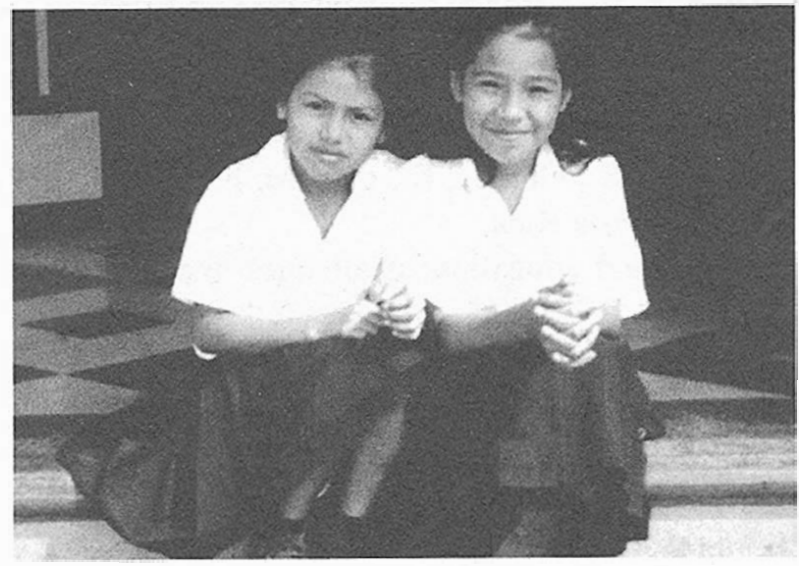

de decirse que tiene derecho a una educación de equidad con una visión de la diversidad como parte inherente al ser humano que debe ser conocida, respetada y atendida. 


\section{REFERENCIAS}

Acuña, G. y Olivares, E. (1999). "La población migrante nicaragüense en Costa Rica: realidad y respuestas". Fundación Arias para la Paz y el Progreso Humano. Recuperado en: http: www.nacion.co.cr/ln_ee/ESPECIALES/nicaragua/paz.html

Alvarenga, P. (1997). "Conflictiva convivencia de los nicaragüenses en Costa Rica". Cuaderno de Ciencias Sociales 101. San José, Costa Rica: FLACSO.

Azofeifa, F., Monge, M., Vidal, M. y Céspedes, E. (2001). Diversidad e integración cultural. Heredia, Costa Rica: CIDE, OIM, MEP, UNA, USAID.

Barth, L. y Díaz, S. (2000). Diversidad e integración cultural. Módulo 1. San José, Costa Rica: Arte e Imagen.

Contreras, V. y Robert, A. (2000). Ampliación de la capacidad del sistema educativo costarricense en comunidades afectadas por la migración nicaragüense relacionada con el huracán Mitch. Costa Rica: Servicios Litográficos Barsant S.A.

Gutiérrez, F. y Prieto, D. (1991). La mediación pedagógica. San José, Costa Rica: RNTC.

James, D. (1997a). "Coping with a new society: the unique psychosocial problems of inmigrant youth". The Journal of School Health, 67, 98-102.

James, D. (1997b). "Psychosocial risks of inmigrant students". The Education Digest, 63, 51-53.

López, M. (s.f.). "La educación intercultural: la diferencia como valor". Recuperado en: http: www.sauce.cnice.mecd.es/smarti4/lopezm.htm

Mata, A., Monge, M., Pereira, T., Alvarado, R., Gómez, K., Cubero, F. y Frías, C. (2000a). Módulo 2.1: Educación de Calidad. Costa Rica.

Mata, A., Monge, M., Pereira, T., Alvarado, R., Gómez, K., Cubero, F. y Frías, C. (2000b). Módulo 2.2: Educar para la diversidad. Costa Rica.

Mata, A., Monge, M., Pereira, T., Alvarado, R., Gómez, K., Cubero, F. y Frías, C. (2000c). Módulo 2.3: Éxito escolar. Costa Rica.

Perkins, L. (2000). "The new inmigrants and education: challenges and issues". Educational Horizons, 78, 67-71.

Porter, E. y Romo, C. (1999). “Abran las puertas!” América Economia, 8 de abril: 37-38.

Savater, F. (2001). Ética para Amador. Madrid: Ariel.

Vásquez, E. (2004). Propuesta didáctica para la capacitación de docentes en la atención a los y las niñas(os) migrantes nicaragüenses de la Escuela Nuevo Horizonte, La Milpa, Heredia. Trabajo final de graduación no publicado, para optar por el título de Maestría en pedagogía con énfasis en la diversidad en los aprendizajes. Universidad Nacional, Heredia, Costa Rica. 